\title{
Micro-mechanisms of residual oil mobilization by viscoelastic fluids
}

\author{
Zhang Lijuan $^{1 *}$, Yue Xiang'an ${ }^{1}$ and Guo Fenqiao ${ }^{2}$ \\ ${ }^{1}$ Key Laboratory of Petroleum Engineering, Ministry of Education, China University of Petroleum, Beijing 102249, China \\ ${ }^{2}$ Development Department, Sinopec Exploration and Production Research Institute, Beijing 100083, China
}

\begin{abstract}
Four typical types of residual oil, residual oil trapped in dead ends, oil ganglia in pore throats, oil at pore corners and oil film adhered to pore walls, were studied. According to main pore structure characteristics and the fundamental morphological features of residual oil, four displacement models for residual oil were proposed, in which pore-scale flow behavior of viscoelastic fluid was analyzed by a numerical method and micro-mechanisms for mobilization of residual oil were discussed. Calculated results indicate that the viscoelastic effect enhances micro displacement efficiency and increases swept volume. For residual oil trapped in dead ends, the flow field of viscoelastic fluid is developed in dead ends more deeply, resulting in more contact with oil by the displacing fluid, and consequently increasing swept volume. In addition, intense viscoelastic vortex has great stress, under which residual oil becomes small oil ganglia, and finally be carried into main channels. For residual oil at pore throats, its displacement mechanisms are similar to the oil trapped in dead ends. Vortices are developed in the depths of the throats and oil ganglia become smaller. Besides, viscoelastic fluid causes higher pressure drop on oil ganglia, as a driving force, which can overcome capillary force, consequently, flow direction can be changed and the displacing fluid enter smaller throats. For oil at pore corners, viscoelastic fluid can enhance displacement efficiency as a result of greater velocity and stress near the corners. For residual oil adhered to pore wall, viscoelastic fluid can provide a greater displacing force on the interface between viscoelastic fluid and oil, thus, making it easier to exceed the minimum interfacial tension for mobilizing the oil film.
\end{abstract}

Key words: Viscoelastic, chemical flooding, displacement mechanism, residual oil, swept volume

\section{Introduction}

Understanding of the mechanisms of polymer flooding has gone through two stages. The earliest idea (Barnes, 1962; Pye, 1964; Sandiford, 1964) had focused on improving macro swept volume. In the $1990 \mathrm{~s}$, different opinions were proposed that viscoelastic polymer solution could enhance not only macro sweep efficiency but also micro displacement efficiency (Han et al, 1995; Mohammad et al, 1992). Much of the prior work was performed on glass micromodels and cores (Wang et al, 2000; 2002). In addition, Daubend and Menzie (1967) and Hester et al (1994) observed when the rate of flow in porous media exceeded a critical value, viscoelastic fluid would produce additional resistance to flow. In recent years, agreement has been made on qualitative understanding of displacement mechanism of viscoelastic polymer solution, which can increase macro swept volume and enhance micro displacement efficiency. However, experimental results are mainly available (Ren et al, 2006) and understanding is merely based on displacement phenomena (Tong et al, 1998; Yang et al, 1999; Kong et al, 2007). There are still some confusion about viscoelastic fluid flow in porous media, and it is necessary to make pore-scale investigation. This paper

* Corresponding author. email: zhangljbj2001@sina.com

Received June 3, 2007 discusses how viscoelastic fluid can mobilize four typical types of waterflood residual oil, and presents its flow behavior in different oil-bearing pores with a numerical method.

\section{Residual oil displacement models}

After water flooding residual oil may exist in different forms, which are related to the structure of porous media, surface wettability, and the properties of oil and water as well as the displacement situation. In general, in water-wet porous media, residual oil exists in the form of ganglia in the middle of pores, or is trapped in pore throats by the Jamin effect. In intermediate wet and oil wet cases, residual oil exists in the form of oil films adhered to large pore walls or oil column trapped in small pores called dead ends or at the corners of pores. Correspondingly, there exist four typical types of residual oil in porous media, residual oil trapped in dead ends, oil ganglia in pore throats, oil at corners of pores and oil film adhered to pore walls. Models of the four types are shown in Fig. 1.

\section{Governing equations}

In chemical flooding, a combination of alkali, surfactant and polymer (ASP) is injected into a reservoir. As far as the rheological properties of chemical oil displacement agents are concerned, they depend on the characteristics of the polymer 

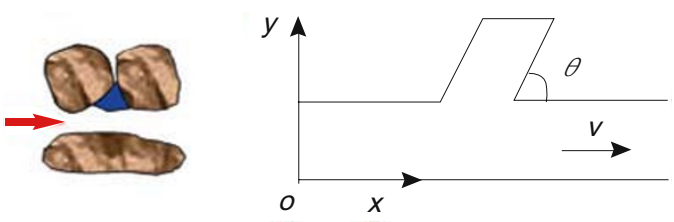

(a) Residual oil in dead ends and its displacement model

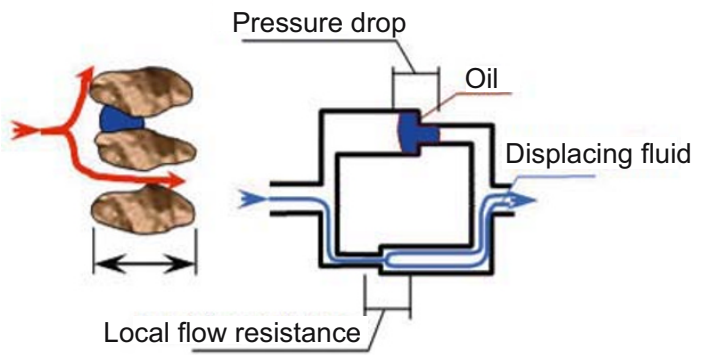

(b) Oil ganglia in throats and its displacement model

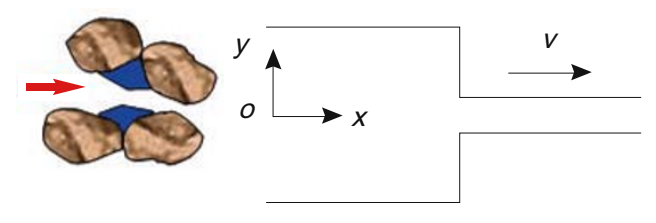

(c) Oil at the corners of pores and its displacement model
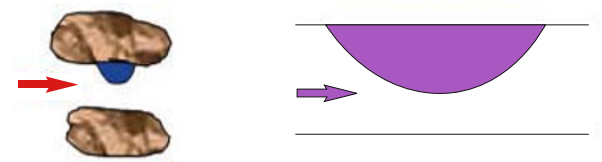

(d) Oil film on pore walls and its displacement model

Fig. 1 Residual oil and its displacement models

solution. In oilfields, partially hydrolyzed polyacrylamide (HPAM) is one of the most widely used polymers, which can be treated as viscoelastic flow in porous media. Assuming that the second normal stress is negligible, the upper-convected Maxwell constitutive equation can be used to describe the rheological behavior of a viscoelastic fluid. The governing equations are as follows.

The steady incompressible plane flow of a viscoelastic fluid is governed by the continuity equation

$$
\frac{\partial u}{\partial x}+\frac{\partial v}{\partial y}=0
$$

The momentum equation

$$
\begin{aligned}
& \rho u \frac{\partial u}{\partial x}+\rho v \frac{\partial u}{\partial y}=-\frac{\partial p}{\partial x}+\frac{\partial T^{x x}}{\partial x}+\frac{\partial T^{x y}}{\partial y} \\
& \rho u \frac{\partial v}{\partial x}+\rho v \frac{\partial v}{\partial y}=-\frac{\partial p}{\partial y}+\frac{\partial T^{x y}}{\partial x}+\frac{\partial T^{y y}}{\partial y}
\end{aligned}
$$

The constitutive equation

$$
\begin{aligned}
& T^{x x}+\lambda\left(u \frac{\partial T^{x x}}{\partial x}+v \frac{\partial T^{x x}}{\partial y}-2 \frac{\partial u}{\partial x} T^{x x}-2 \frac{\partial u}{\partial y} T^{x y}\right)=2 \eta \frac{\partial u}{\partial x} \\
& T^{x y}+\lambda\left(u \frac{\partial T^{x y}}{\partial x}+v \frac{\partial T^{x y}}{\partial y}-\frac{\partial u}{\partial y} T^{y y}-\frac{\partial v}{\partial x} T^{x x}\right) \\
& =\eta\left(\frac{\partial u}{\partial y}+\frac{\partial v}{\partial x}\right)
\end{aligned}
$$

$T^{y y}+\lambda\left(u \frac{\partial T^{y y}}{\partial x}+v \frac{\partial T^{y y}}{\partial y}-2 \frac{\partial v}{\partial x} T^{x y}-2 \frac{\partial v}{\partial y} T^{y y}\right)=2 \eta \frac{\partial v}{\partial y}$

where $u$ and $v$ denote velocity in the $x$ and $y$ direction, respectively; $p$ denotes pressure; $T^{x y}$ denotes shear stress; $T^{x x}$ and $T^{y y}$ denote normal stresses; $\rho$ denotes density; $\lambda$ denotes relaxation time; $\eta$ denotes zero-shear viscosity.

The finite difference method used is essentially that of Cochrane et al (1982), with the modification of non-uniform mesh size. Eqs. (1) through (5) are written in stream functionvorticity formulation with the pressure variable eliminated. The nonlinearly coupled matrix systems are solved in turn by using under-relaxation iteration and Gauss-Seidel iteration.

\section{Results and discussion}

A comparison was made between numerical and experimental results for the development of velocity profiles of HPAM solution in the main flow direction (Wunderlich et al, 1988), as shown in Fig. 2. This illustrates that the upperconvected Maxwell constitutive equation can be used to describe the flow behavior of HPAM solution in reservoir pores. In Fig. 2, Weissenberg number, We is a dimensionless parameter, measuring the magnitude of viscoelasticity, the larger the $W e$, the greater the viscoelasticity.

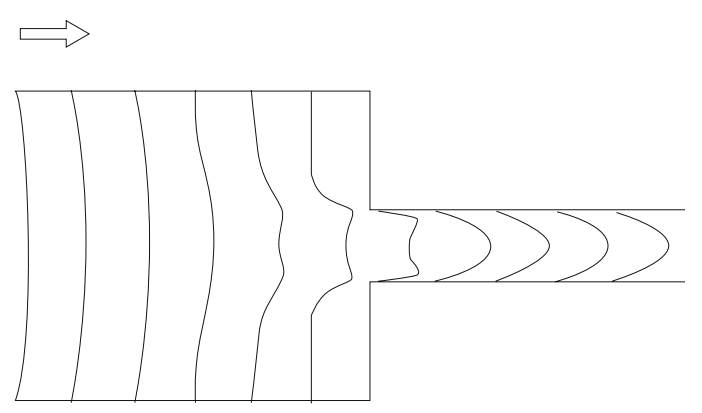

(a) $R e=10, W e=0.4$

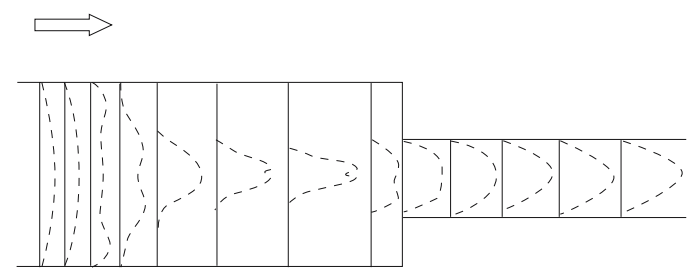

(b) HPAM $C \mathrm{p}=50 \mathrm{mg} / \mathrm{L}, R e=200$

Fig. 2 A comparison between numerical and experimental results

\subsection{Residual oil in dead ends}

It is well known that to mobilize the residual oil the essential premise is that displacing fluids reach the positions where residual oil is trapped. Flow fields of viscoelastic polymer solution, as shown in Fig. 3, indicates that an intense vortex appears in the dead end and is developed downwards when a viscoelastic fluid flows through a pore with a dead end, compared with a viscous fluid. In addition, Fig. 4 also illustrates that viscoelastic fluid has greater stress than viscous fluid.

There are two important mechanisms of mobilization of oil in dead ends displaced by viscoelastic polymer solution. 


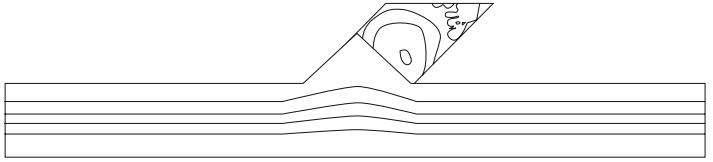

(a) $W e=0, \theta=45^{\circ}$

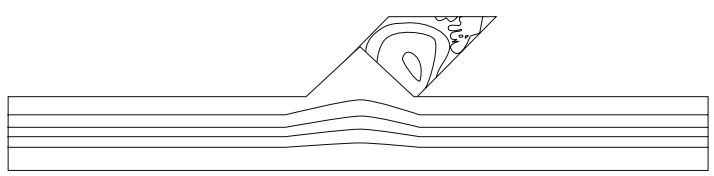

(b) $W e=0.2, \theta=45^{\circ}$

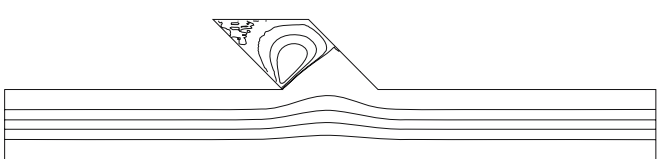

(c) $W e=0, \theta=135^{\circ}$

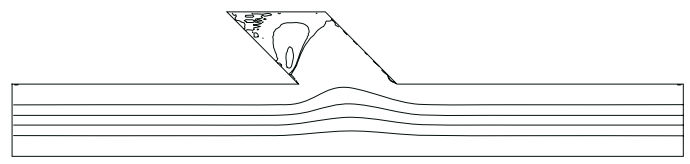

(d) $W e=0.2, \theta=135^{\circ}$

Fig. 3 Flow fields in pores with a dead end

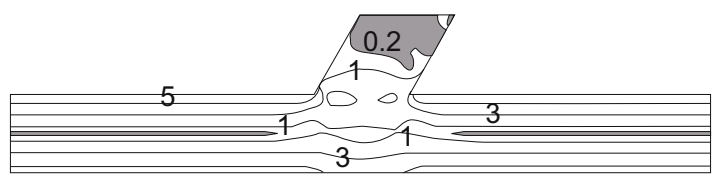

(a) $W e=0, \theta=60^{\circ}$

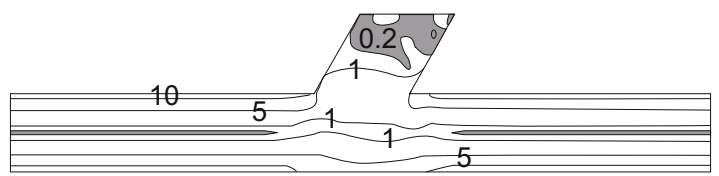

(b) $\mathrm{We}=0.2, \theta=60^{\circ}$

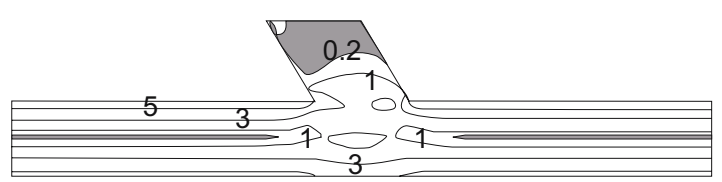

(c) $W e=0, \theta=120^{\circ}$

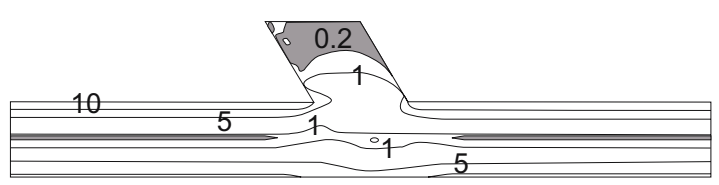

(d) $W e=0.2, \theta=120^{\circ}$

Fig. 4 Contour lines of modulus of deviatoric stress tensor in pores with dead end $(R e=0.001)$
One is that the viscoelastic vortex is developed more deeply to contact more oil; the other is that the viscoelastic vortex, which is of greater stress, disperses residual oil into movable oil ganglia, and finally carries them into the main channels.

\subsection{Oil ganglia in pore throats}

For the mobilization of oil ganglia trapped in pore throats, the displacement mechanisms are similar to residual oil trapped in dead ends. Strong vortices appear and develop near the pore throats, resulting in the mobilization of oil ganglia trapped in pore media, as shown in Fig. 5. Besides, viscoelastic fluid can provide a greater displacing force (generated by pressure drop of viscoelastic fluid), which can counteract the trapping capillary force and so facilitate flow into smaller pores. Usually, the above displacement mechanisms exist when the displacing fluid flows in pore throats. If the oil ganglia are larger or the pressure drop applied on them is smaller, residual oil is mobilized by flowinduced vortex, oil ganglia become gradually smaller and smaller. On the contrary, oil ganglia are mobilized by the displacing force, in which displacement efficiency depends obviously on two factors, interfacial tension, and displacing force. This demonstrates the comprehensive effect of displacing fluid/oil interfacial properties and rheological properties of the displacing fluid.

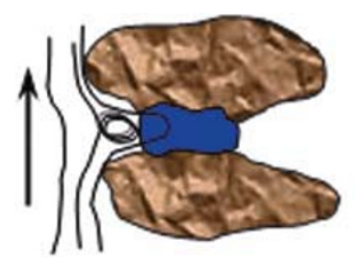

Fig. 5 Displacement of oil ganglia in pore throats

Governing equations (1) through (6) were used to calculate the flow resistance to viscoelastic fluid flow at the position where the cross section is changed (Zhang et al, 2005). Fig. 6 shows that the local resistance to viscoelastic fluid flow at the throat entrance is higher than that to viscous fluid flow. The stronger the viscoelasticity, the greater the local flow resistance. Viscoelastic fluid flow is favorable to generating greater pressure drop on oil ganglia as the displacing force.

\subsection{Oil at the corners of pores}

Fig. 7 shows iso-velocity contour in constricted channels, with a ratio of pore to throat of 4 . The contoured velocities of $0.05,0.025$, and 0.125 shift to the corner with increasing $W e$. This illustrates that the velocity of viscoelastic fluid near the corner is higher than that of viscous fluid. In addition, as shown Fig. 8, viscoelastic fluid has greater stress than viscous fluid. With large velocity and displacing force, viscoelastic fluid can enhance micro displacement efficiency of oil trapped at the corners of pores.

\subsection{Oil film on pore walls}

Oil film on pore walls usually refers to a adsorbed layer of 


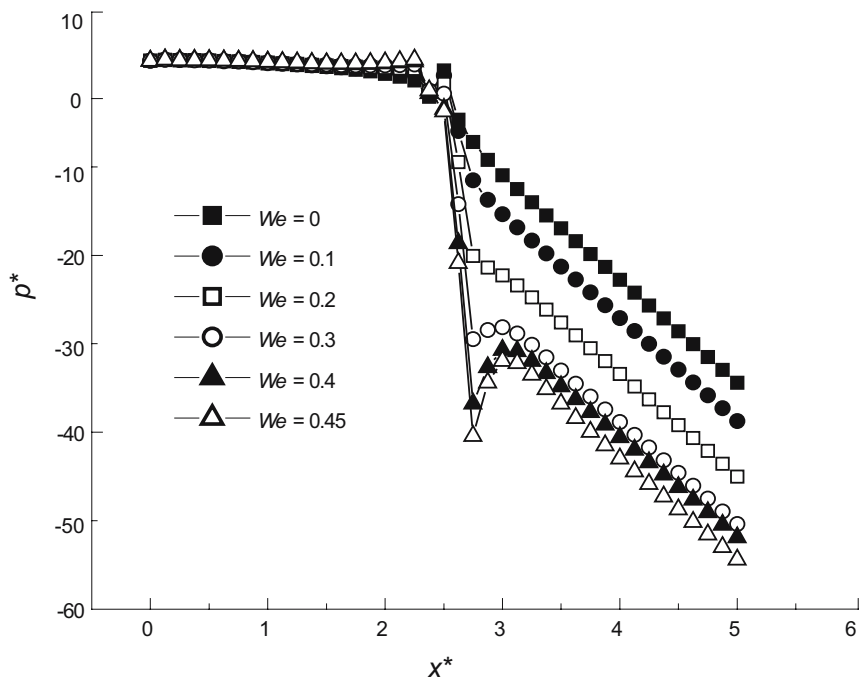

(a) Constricted channel

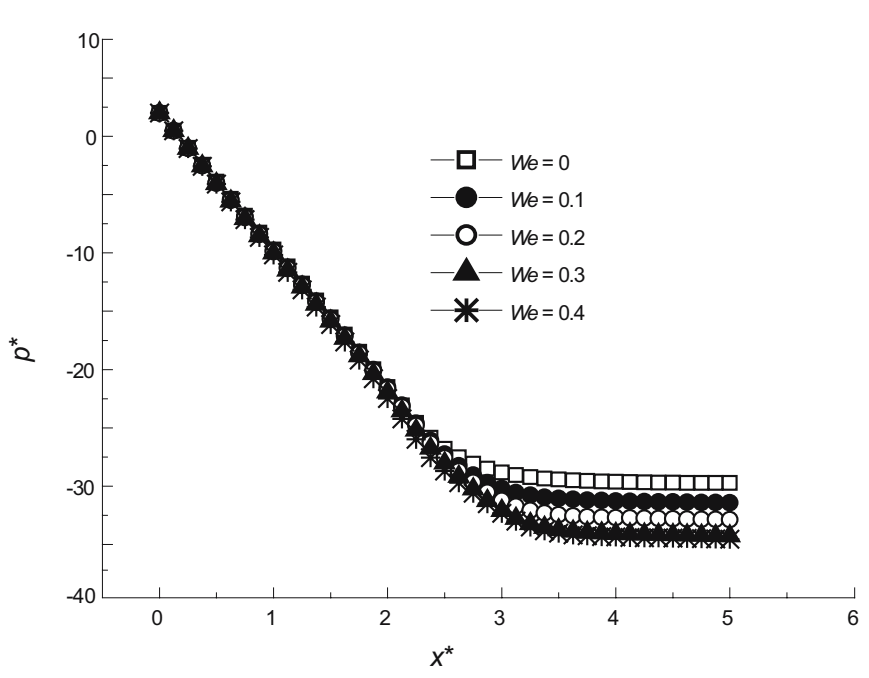

(b) Expanded channel

Fig. 6 Pressure distribution in varied cross-sectional channels, with a ratio of pore to throat of 4

varying thickness or oil ganglia adhered on pore walls. Due to the effects of crude oil/rock interfacial properties, the oil film on pore walls is characterized by yield stress, which varies along the direction vertical to the pore wall. In steady planar flow, as shown in Fig. 1(d), the oil film constitutive equation is as follows:

$$
\begin{cases}\dot{\gamma}=0 & \tau \leq \tau_{y} \\ \tau-\tau_{y}=\eta_{p} \dot{\gamma} & \tau>\tau_{y}\end{cases}
$$

where $\dot{y}$ is shear rate, $\mathrm{s}^{-1} ; \quad \tau$ is shear stress, $\mathrm{Pa}, \tau_{y}$ is yield stress, Pa.

According to the relationship curve of residual oil film versus displacing pressure gradient measured by the centrifuge method(Мархасин and Рстрокина, 1973), the relationship between yield stress and the distance away from pore wall can be obtained as follows:

$$
\tau_{y}=\tau_{0}\left(\frac{\varepsilon D}{D_{\mathrm{P}}-y+a_{0} D_{\mathrm{P}}}\right)^{n^{\prime}}
$$

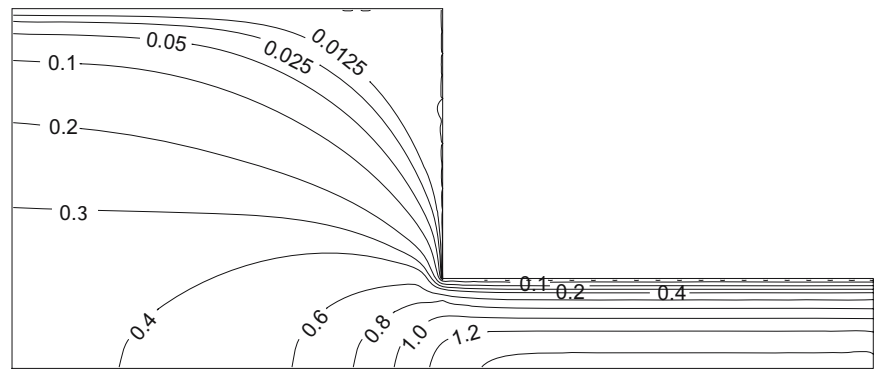

(a) $W e=0$

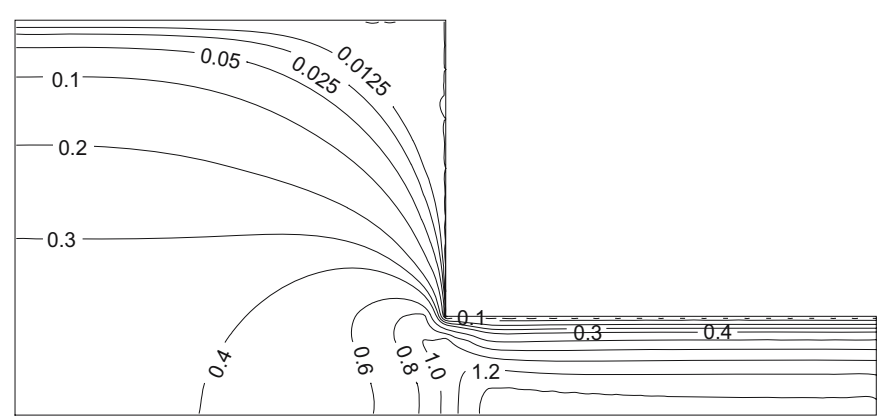

(b) $W e=0.4$

Fig. 7 Iso-velocity contour in constricted channels $(R e=0.001)$

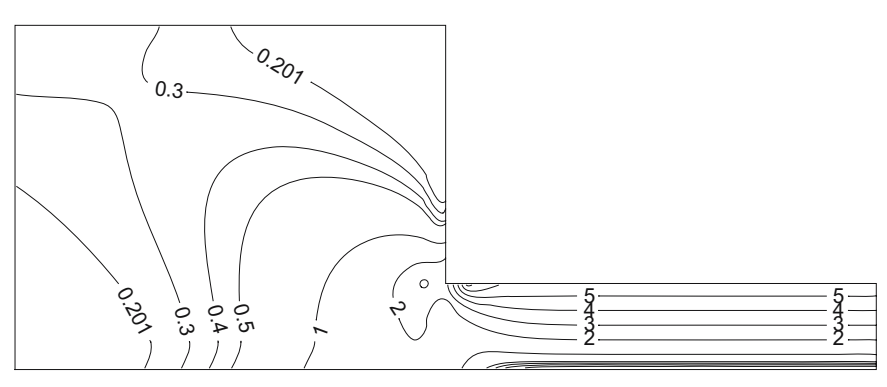

(a) $W e=0$

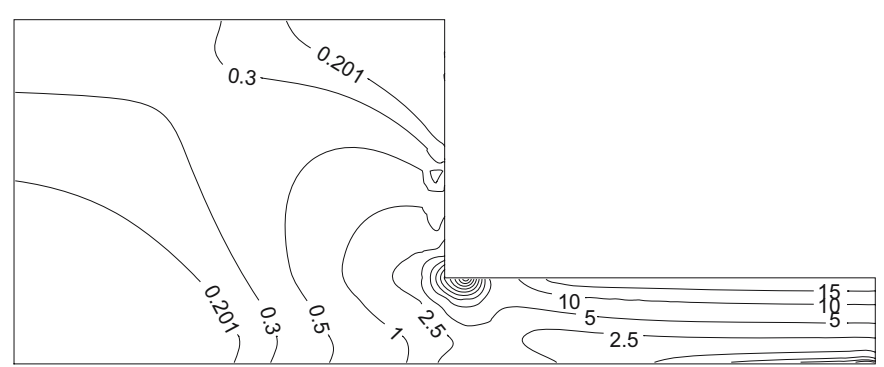

(b) $W e=0.4$

Fig. 8 Contour lines of modulus of deviatoric stress tensor in constricted channels $(R e=0.001)$

where, $\tau_{0}$ denotes the yield stress at the interface between oil and displacing fluid, $\mathrm{Pa} ; D$ is the distance from the central oil film to the wall, $\mu \mathrm{m} ; \quad \varepsilon$ is a ratio of the central oil film thickness to $D ; D_{\mathrm{p}}$ is the pore diameter, $D_{\mathrm{P}}=(1+\varepsilon) D, \mu \mathrm{m} ; y$ is any point on oil film, $\mu \mathrm{m} ; a_{0}$ and $n^{\prime}$ are constants, dependent on the physicochemical properties of the pore wall and crude oil.

Yield stress, as a function of distance of oil film away from pore wall, was calculated by Eq. (8) and is shown in 
Fig. 9, for $n^{\prime}=4, a_{0}=0.0001, \varepsilon=1.5$.

Given that oil film and pore system are multiphase and multi-interface, the displacement of oil film occurs firstly at

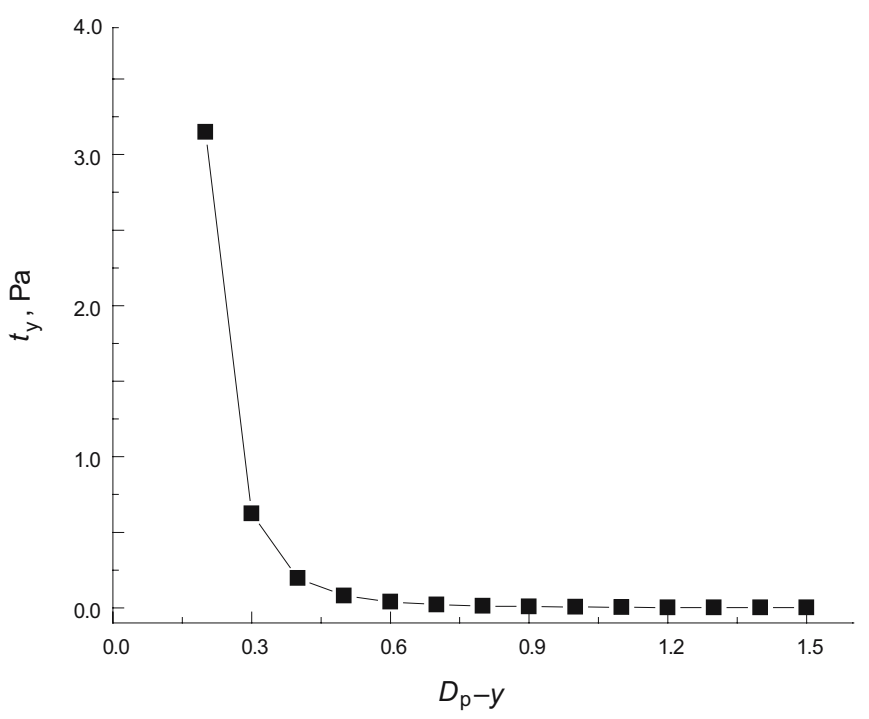

Fig. 9 Yield stress as a function of the distance of oil film away from the pore wall

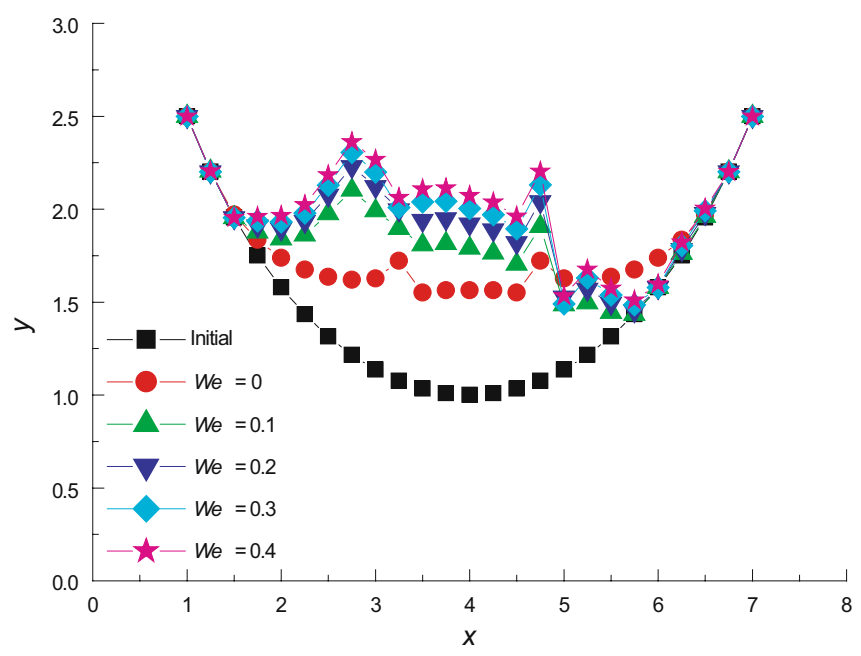

(a) $\sigma=1 \times 10^{-3} \mathrm{mN} / \mathrm{m}, \varepsilon=1.5$

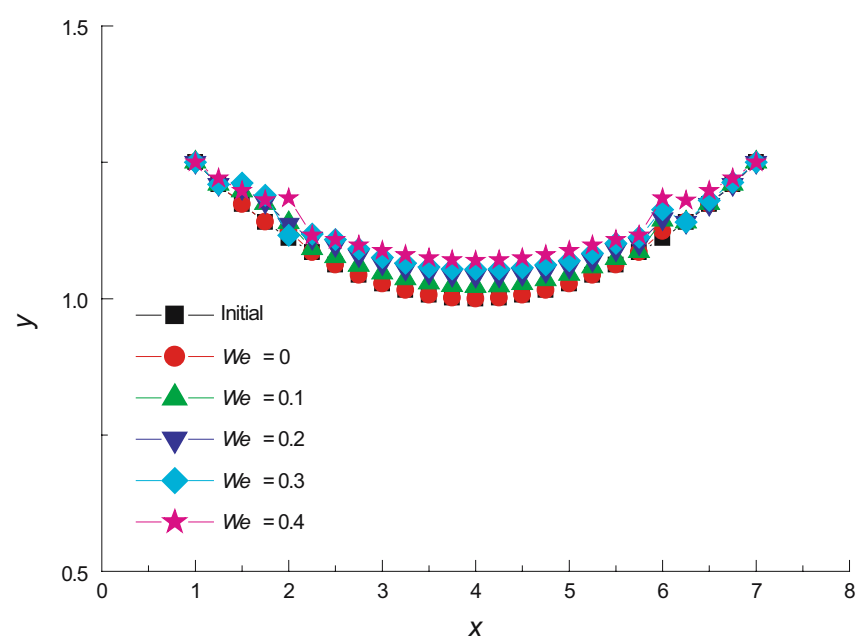

(c) $\sigma=1 \times 10^{-3} \mathrm{mN} / \mathrm{m}, \varepsilon=2.5$ the displacing fluid/oil interface. The process is described as follows, the above-mentioned displacing force deforms the oil film, and part of the oil is entrained into the main stream, then the rest of the oil film becomes thin. When the displacing force increases or interfacial force decreases, the oil film becomes thinner, and finally it is removed. However, a resistance force, arising from interfacial force and yield stress of the oil film, tends to prevent the oil film from deforming and flowing. The essential premise for partially mobilizing the oil film is that the displacing force is larger than the restraining force.

Eqs. (1) through (6) were used to calculate the flow behavior of viscoelastic fluid displacing an oil film adhered to pore walls (as shown in Fig. 1(d)). Combined with the above-mentioned essential premise for oil film displacement, the oil film shape in the displacement process is depicted in Fig. 10. For the same crude oil, decreasing interfacial tension or enhancing the viscoelasticity of the displacing fluid could enhance the displacement efficiency of oil film. In Fig. 10, $x$, $y, \varepsilon$ are dimensionless; $v$ is the flow rate, $\mathrm{m} / \mathrm{d}$; and $\sigma$ is the interfacial tension, $\mathrm{mN} / \mathrm{m}$.

Fig. $10(\mathrm{c}, \mathrm{d})$ shows that there is a start-up interfacial

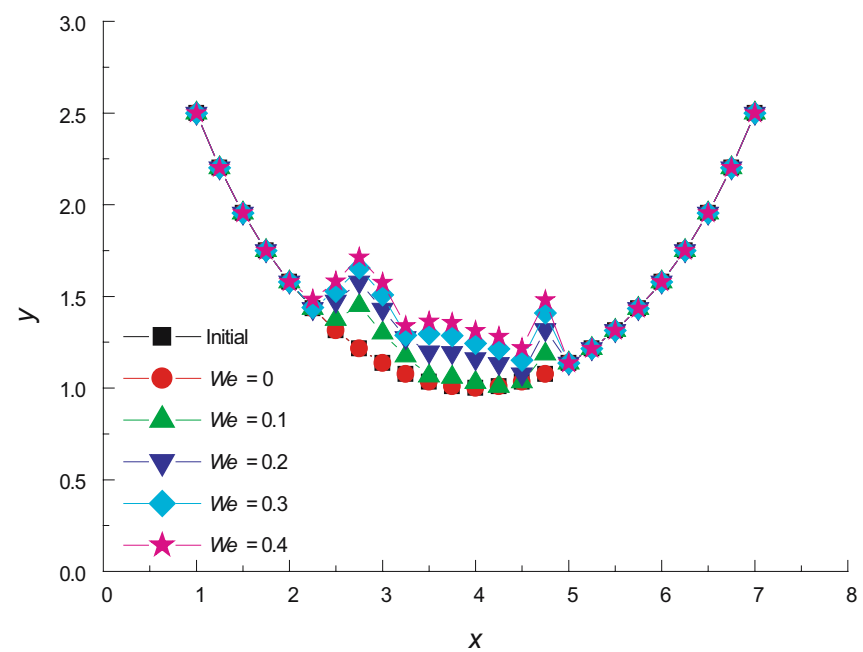

(b) $\sigma=1 \times 10^{-2} \mathrm{mN} / \mathrm{m}, \varepsilon=1.5$

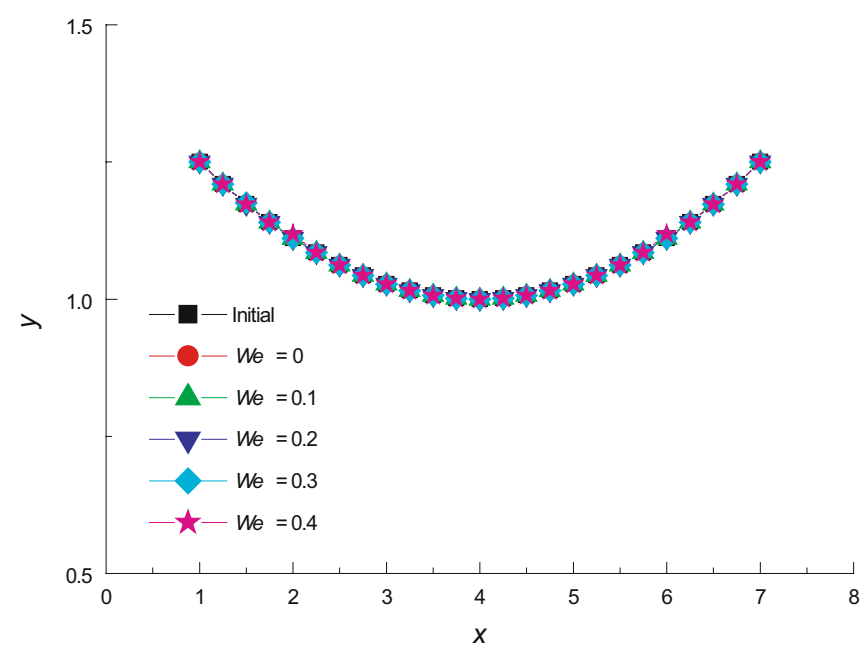

(d) $\sigma=1 \times 10^{-2} \mathrm{mN} / \mathrm{m}, \varepsilon=2.5$

Fig. 10 Oil film shape when displaced by fluid with varied viscoelasticity ( $\eta=10 \mathrm{mPa} \cdot \mathrm{s}, \nu=10 \mathrm{~m} / \mathrm{d}, D=40 \mu \mathrm{m})$ 
tension for mobilization of an oil film, which is the lowest interfacial tension for displacement of some of an oil film adhered to given pore walls. For a thin oil film $(\varepsilon=0.25)$, even if the interfacial tension is lowered to $1 \times 10^{-3} \mathrm{mN} / \mathrm{m}$, a viscous fluid can not mobilize the oil film; however, a viscoelastic fluid can mobilize some of the oil film but cannot do so when the interfacial tension increases to $1 \times 10^{-2} \mathrm{mN} / \mathrm{m}$. This indicates that only when start-up interfacial tension is reached, can an oil film be partially displaced. The thickness of the oil film and fluid viscoelasticity have important effects on start-up interfacial tension. Assuming oil film is much thicker $(\varepsilon=1.5)$, as shown in Fig. 10(b), for interfacial tension of $1 \times 10^{-2} \mathrm{mN} / \mathrm{m}$, a viscoelastic fluid can still mobilize some of the oil film, while a viscous fluid $(W e=0)$ can not do so. Because a viscoelastic fluid can provide larger displacing force applying on the interface between viscoelastic fluid and oil, thus, with an appropriate increase in viscoelasticity, an ultra-low interfacial tension for the thin oil film is not needed.

\section{Conclusions}

In polymer flooding to enhance recovery of residual oil, the viscoelastic effect enhances micro displacement efficiency and increases swept volume. Viscoelastic fluid provides greater stress and velocity at the changed section of pores, which help disperse and entrain residual oil. In addition, it generates a greater pressure drop (as a displacing force), and stronger vortices to increase micro swept volume of smaller pores and dead end.

\section{Acknowledgements}

This paper was supported by National Natural Science Foundation of China (Grant No: 50574060) and National Basic Research Program of China (Grant No: 2005CB221300).

\section{References}

Barnes A L. The use of a viscous slug to improve waterflood efficiency in a reservoir partially invaded by bottom water. JPT. 1962. 14(10):1147-1153

Cochrane T, Walters K and Webster M F. Newtonian and non-Newtonian flow near a reentrant corner. J. Non-Newt. Fluid Mech. 1982. 10: 95-114

Daubend Jr D L and Menzie D E. Flow of polymer solutions through porous media. JPT. 1967. 19(8): 1065-1073

Han X Q, Wang W Y and Xu Y. The viscoelastic behavior of HPAM solutions in porous media and its effects on displacement efficiency. International Meeting on Petroleum Engineering held in Beijing, China, 14-17 November, 1995 (SPE paper 30013)

Hester R D, Flesher L M and McCormick C L. Polymer solution extension viscosity effects during reservoir flooding. SPE/DOE Improved Oil Recovery Symposium held in Tulsa, Oklahoma, USA, 17-20 April, 1994 (SPE/DOE paper 27823)

Kong F S, Wang X L and Xia H F. Microcosmic displacement mechanism of viscoelastic polymer solution displacing the residual oil at the dead end. Petroleum Geology \& Oilfield Development in Daqing. 2007. 20(5): 110-113 (in Chinese)

Мархасин И Л and Рстрокина В. Методика И Результаты Послойного Изучения Граничного Слоя Нефти На Контакте С Твердой Поверхностью. Нефть И Газ. 1973. (5): 83-86 (in Russian)

Mohammad R, Juergen R, Guenter P, et al. Quantification and optimization of viscoelastic effects of polymer solutions for enhanced oil recovery. SPE/DOE Enhanced Oil Recovery Symposium held in Tulsa, Oklahoma, USA, 22-24 April, 1992 (SPE/DOE paper 24154)

Pye D J. Improved secondary recovery by control of water mobility. JPT. 1964. 16(8): 911-916

Ren K, Wang J L, Lin M Q, et al. Rheological properties of hydrophobically associating polyacrylamide solution. Petroleum Science. 2006. 3(2): 66-72

Sandiford B B. Laboratory and field studies of water floods using polymer solutions to increase oil recoveries. JPT. 1964. 16(8): 917-922

Tong Z S, Yang C Z, Wu G Q, et al. A study of microscopic flooding mechanism of surfactant/alkali/polymer. SPE/DOE Improved Oil Recovery Symposium held in Tulsa, Oklahoma, USA, 19-22 April, 1998 (SPE/DOE paper 39662)

Wang D M, Cheng J C, Xia H F, et al. Improvement of displacement efficiency of cores by driving forces parallel to the oil-water interface of viscous-elastic fluids. Acta Petrolei Sinica. 2002. 23(5): 48-52 (in Chinese)

Wang D M, Cheng J C, Yang Q Y, et al. Viscous-elastic polymer can increase microsclae displacement efficiency in cores. SPE Annual Technical Conference and Exhibition held in Dallas, Texas, USA, 1-4 October, 2000 (SPE paper 63227)

Wunderlich A M, Brunn P O and Durst F. Flow of dilute polyacrylamide solutions through a sudden planar contraction. J. Non-Newt. Fluid Mech. 1988. 28: 267-285

Yang Q Y, Gong W C and Jia Z W. Experimental study on oil displacement efficiency of produced fluids and reformulated system of ASP flooding. Petroleum Geology \& Oilfield Development in Daqing. 1999. 18(3): 24-26 (in Chinese)

Zhang L J, Yue X A, Liu Z C, et al. Percolation mechanism of polymer solution through porous media. Journal of the University of Petroleum, China (Edition of Natural science). 2005. 29(1): 51-55 (in Chinese)

(Edited by Sun Yanhua) 